\title{
High Catalytic Activity and Chemoselectivity of Sub-Nanometric Pd Clusters on Porous Nanorods of $\mathrm{CeO}_{2}$ for Hydrogenation of Nitroarenes
}

\author{
Sai Zhang, ${ }^{\dagger}$ Chun-Ran Chang, ${ }^{\ddagger}$ Zheng-Qing Huang, ${ }^{\ddagger}$ Jing Li, ${ }^{\dagger}$ Zhemin Wu, ${ }^{\#}$ \\ Yuanyuan Ma, ${ }^{\dagger}$ Zhiyun Zhang, ${ }^{\dagger}$ Yong Wang ${ }^{\#}$ and Yongquan $\mathrm{Qu}^{*}{ }^{\dagger}, \S$ \\ ${ }^{\dagger}$ Center for Applied Chemical Research, Frontier Institute of Science and Technology \\ and State Key Laboratory for Mechanical Behavior of Materials, Xi'an Jiaotong \\ University, Xi'an, 710049, China \\ ${ }^{*}$ Institute of Industrial Catalysis, School of Chemical Engineering and Technology, \\ Xi'an Jiaotong University, Xi'an, 710049, China \\ \# School of Material Science and Engineering, Centre of Electron Microscopy and \\ State Key Laboratory of Silicon Materials, Zhejiang University, Hangzhou, 310028, \\ China \\ ${ }^{\S}$ MOE Key Laboratory for Nonequilibrium Synthesis and Modulation of Condensed \\ Matter, Xi'an Jiaotong University, Xi'an, 710049, China
}

*E-mail: yongquan@mail.xjtu.edu.cn. 

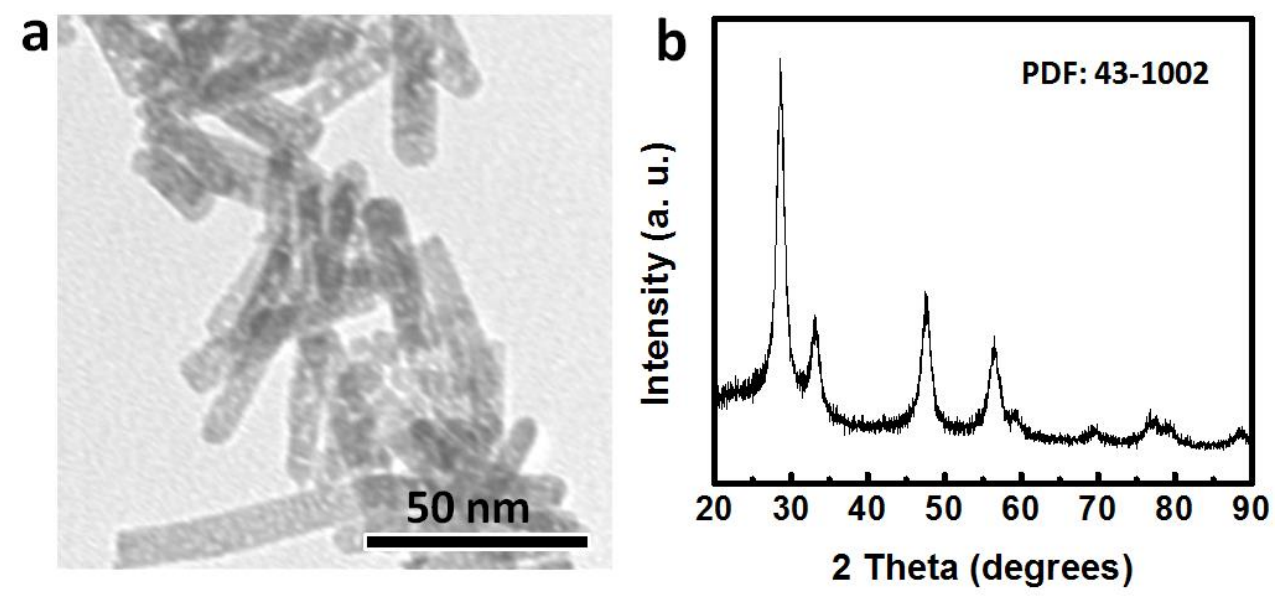

Figure S1. TEM image and XRD spectrum of $P N-\mathrm{CeO}_{2}$.

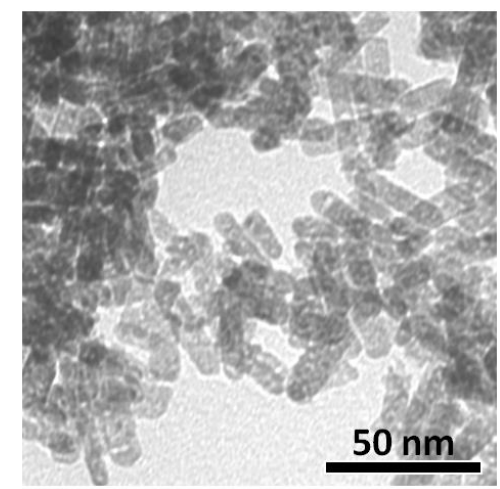

Figure S2. Bright field TEM image of $\mathrm{PdsNC} / P N-\mathrm{CeO}_{2}$ catalysts. 

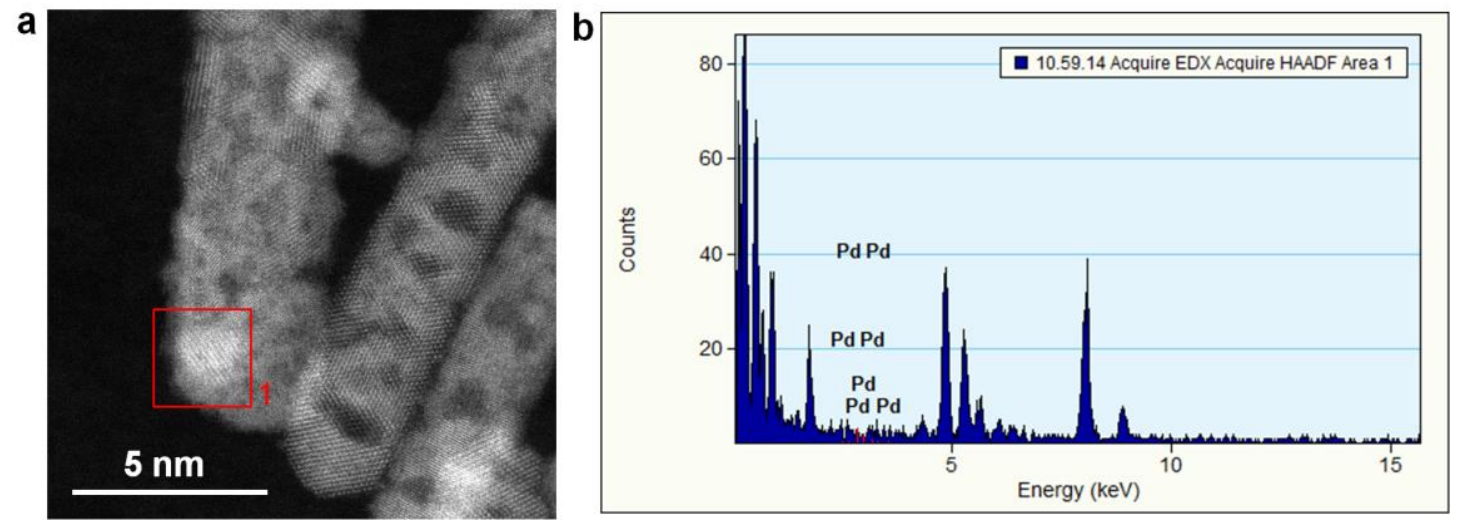

Figure S3. The EDS spectrum of $\mathrm{Pd}$ element on $\mathrm{PdsNC} / \mathrm{PN}-\mathrm{CeO}_{2}$ catalysts. 

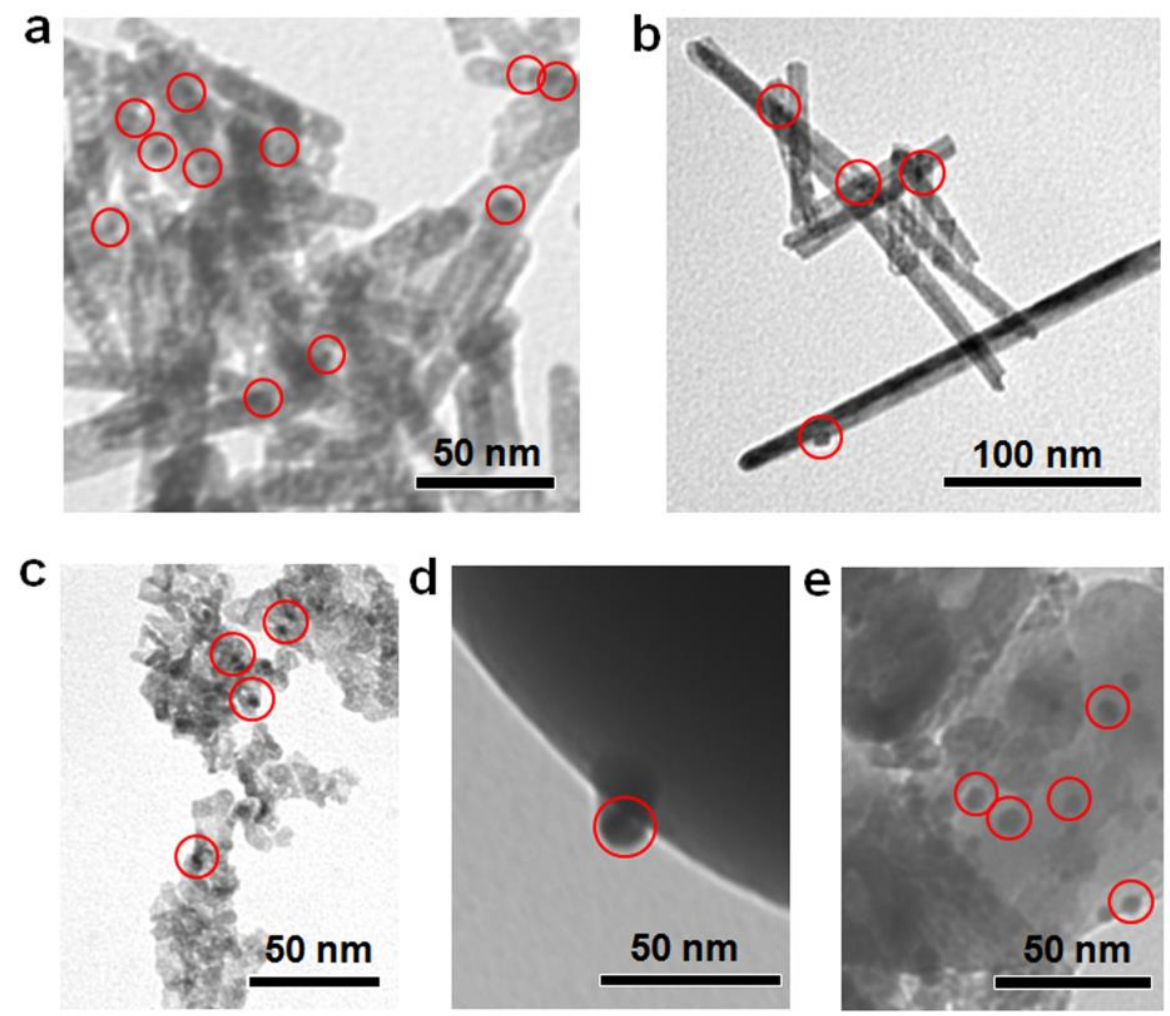

Figure S4. TEM images of (a) $\mathrm{PdNP} / P N-\mathrm{CeO}_{2}$, (b) $\mathrm{PdNP} / N R-\mathrm{CeO}_{2}$, (c) $\mathrm{PdNP} / \mathrm{NP}-\mathrm{CeO}_{2}$, (d) $\mathrm{PdNP} / \mathrm{SiO}_{2}$ and (e) $\mathrm{PdNP} / \mathrm{TiO}_{2}$ catalysts. 
a

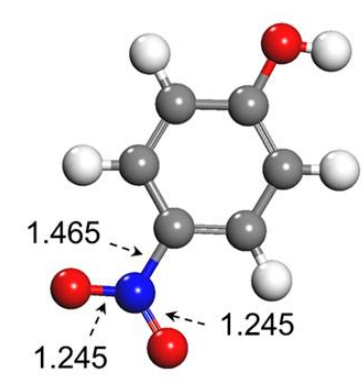

b

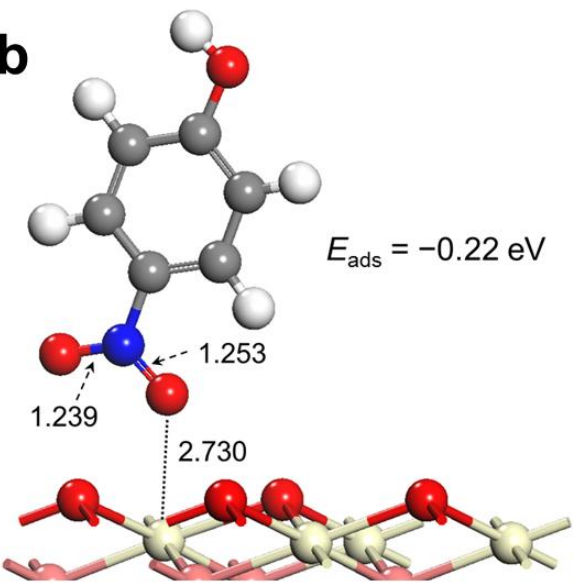

Figure S5. (a) The optimized structure and bond distances $(\AA)$ of 4-nitrophenol and (b) the adsorption model of 4-nitrophenol on the ideal (111) crystal face of $\mathrm{CeO}_{2}$. The adsorption energy and bond distances $(\AA)$ are labeled in figure. 

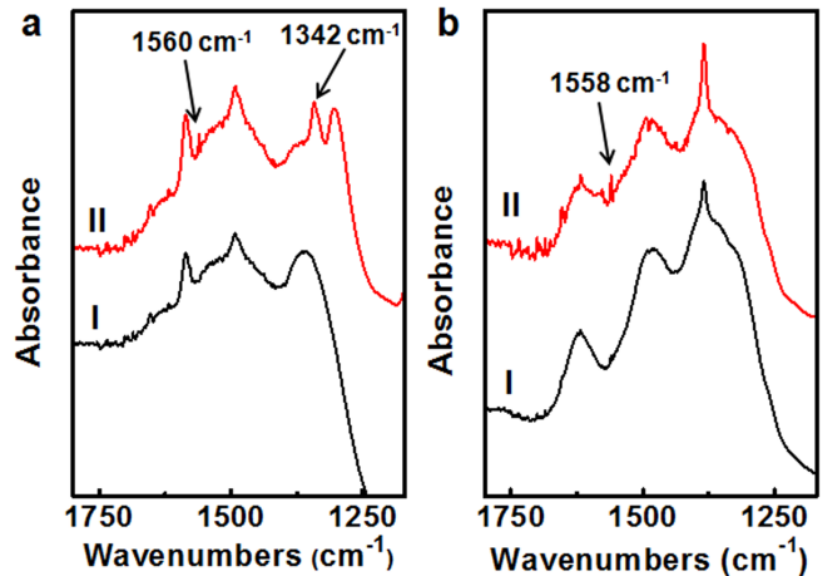

Figure S6. FTIR spectra of (a) $N R-\mathrm{CeO}_{2}$ and (b) $N P-\mathrm{CeO}_{2}$ before (I) and after (II) adsorption of 4-nitrophenol, respectively. 

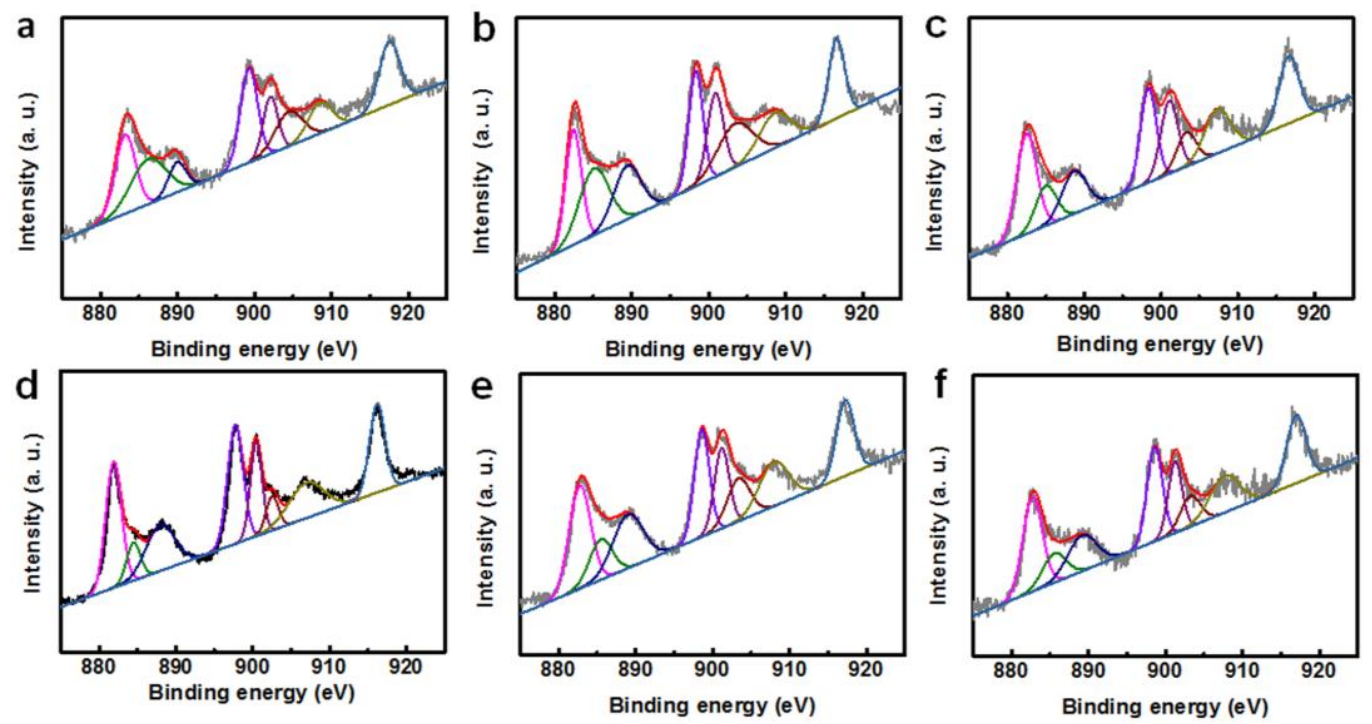

Figure S7. The XPS spectrum of (a) $\mathrm{PdsNC} / P N-\mathrm{CeO}_{2}$, (b) $\mathrm{PdNP} / P N-\mathrm{CeO}_{2}$, (c) $\mathrm{PdNP} / N R-\mathrm{CeO}_{2}$, (d) $\mathrm{PdNP} / \mathrm{NP}-\mathrm{CeO}_{2}$, (e) $\mathrm{PdsNC} / P N-\mathrm{CeO}_{2}-300$ and (f) $\mathrm{PdsNC} / P N-\mathrm{CeO}_{2}-500$ catalysts. 

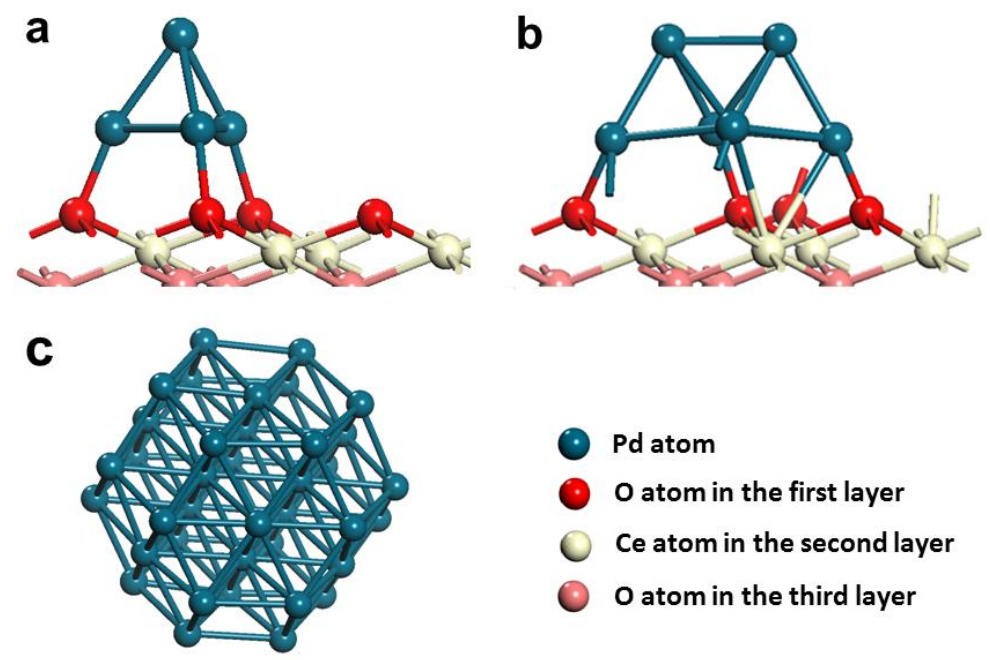

Figure S8. The calculation model of (a) $\mathrm{Pd}_{4} / P N-\mathrm{CeO}_{2}$, (b) $\mathrm{Pd}_{6} / P N-\mathrm{CeO}_{2}$ and (c) $\mathrm{Pd}_{38}$ nanoparticle. 

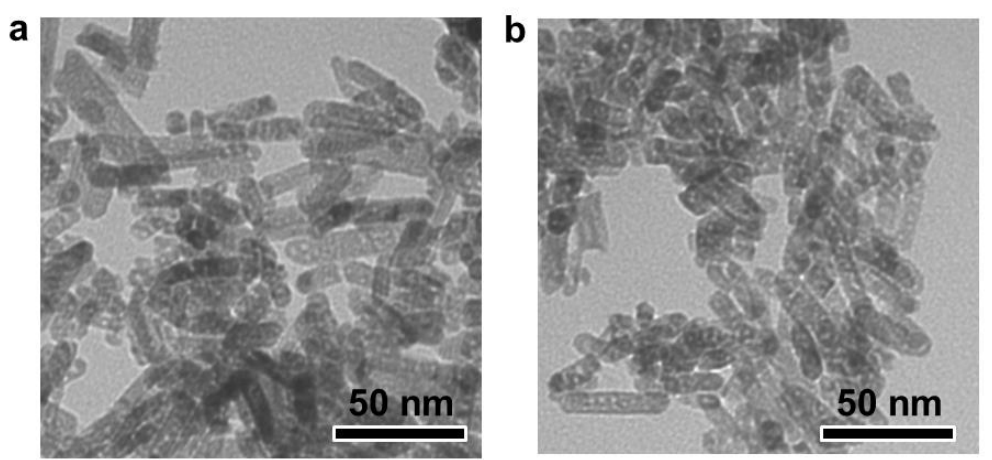

Figure S9. TEM iamges of (a) $\mathrm{PdsNC} / P N-\mathrm{CeO}_{2}-300$ and (b) $\mathrm{PdsNC} / P N-\mathrm{CeO}_{2}-500$ catalysts. 

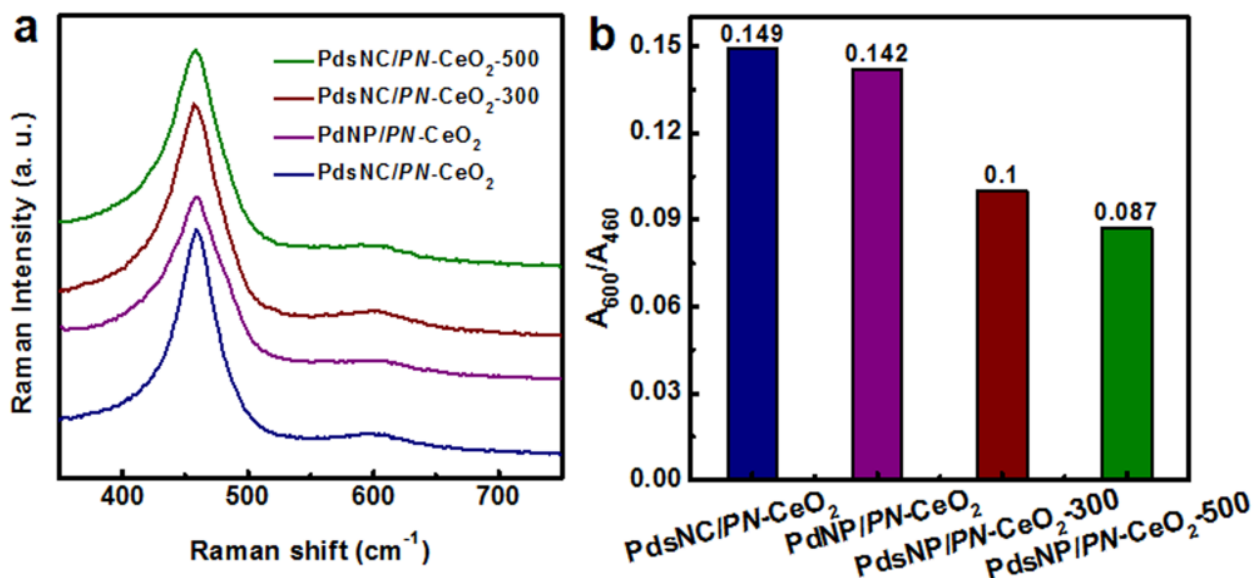

Figure S10. (a) Raman spectra and (b) plot of the ratio of $\mathrm{A}_{600} / \mathrm{A}_{460}$ of various catalysts. 


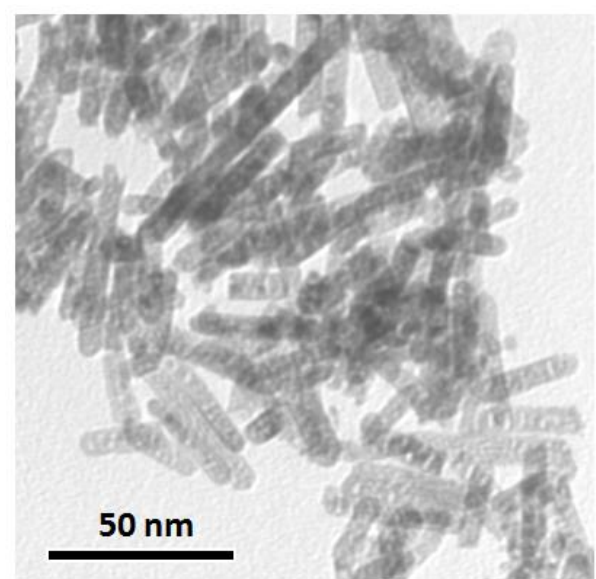

Figure S11. TEM image of the high dispersion $\mathrm{PdsNC} / P N-\mathrm{CeO}_{2}$ catalysts after 6 cycles of catalytic reactions. 
Table S1. The optimization of reaction condition for hydrogenation of 4-nitrophenol*

\begin{tabular}{|c|c|c|c|c|c|c|}
\hline Entry & Solvent & $\begin{array}{l}\text { Time } \\
(\min )\end{array}$ & $\begin{array}{c}\text { Temperature } \\
\left({ }^{\circ} \mathrm{C}\right)\end{array}$ & $\begin{array}{c}\text { Pressure } \\
\text { (MPa) }\end{array}$ & $\begin{array}{c}\text { Conversion } \\
(\%)\end{array}$ & $\begin{array}{c}\text { Selectivity } \\
(\%)\end{array}$ \\
\hline 1 & $\mathrm{MeOH}$ & 60 & 80 & 1 & 72.5 & $>99.9$ \\
\hline 2 & Ethanol & 60 & 80 & 1 & 58.7 & $>99.9$ \\
\hline 3 & $\mathrm{H}_{2} \mathrm{O}$ & 60 & 80 & 1 & 77.8 & 95.9 \\
\hline 4 & THF & 60 & 80 & 1 & 36.2 & $>99.9$ \\
\hline 5 & $\mathrm{MeOH}$ & 60 & 90 & 1 & 98.7 & $>99.9$ \\
\hline 6 & $\mathrm{MeOH}$ & 60 & 70 & 1 & 56.6 & $>99.9$ \\
\hline 7 & $\mathrm{MeOH}$ & 60 & 80 & 2 & $>99.9$ & $>99.9$ \\
\hline 8 & $\mathrm{MeOH}$ & 60 & 80 & 1.5 & $>99.9$ & $>99.9$ \\
\hline 9 & $\mathrm{MeOH}$ & 60 & 80 & 0.5 & 58.7 & $>99.9$ \\
\hline 10 & $\mathrm{MeOH}$ & 30 & 80 & 0.5 & 30.5 & $>99.9$ \\
\hline 11 & $\mathrm{MeOH}$ & 90 & 80 & 0.5 & 75.3 & $>99.9$ \\
\hline 12 & $\mathrm{MeOH}$ & 120 & 80 & 0.5 & $>99.9$ & $>99.9$ \\
\hline 13 & $\mathrm{MeOH}$ & 30 & 120 & 0.5 & $>99.9$ & $>99.9$ \\
\hline 14 & $\mathrm{MeOH}$ & 10 & 120 & 0.5 & 41.3 & $>99.9$ \\
\hline $15^{\mathrm{a}}$ & $\mathrm{MeOH}$ & 120 & 80 & 0.5 & 0 & - \\
\hline $16^{\mathrm{a}}$ & $\mathrm{MeOH}$ & 600 & 80 & 0.5 & 0 & - \\
\hline
\end{tabular}

* Reaction condition: $5 \mathrm{mg}$ high dispersion $\mathrm{PdsNC} / P N-\mathrm{CeO}_{2}$ catalysts, $0.5 \mathrm{mmol}$ of 4-nitrophenol and $4 \mathrm{~mL}$ of solvent.

${ }^{\text {a }} 5 \mathrm{mg} P \mathrm{~N}-\mathrm{CeO}_{2}$ catalysts. 
Table S2. Summary of parameters of various catalysts for hydrogenation 4-nitrophenol.

\begin{tabular}{ccccc}
\hline Catalysts & $\begin{array}{c}\mathbf{C e}^{\mathbf{3 +}} \text { of } \\
\text { support }\end{array}$ & $\begin{array}{c}\text { Dispersion of Pd } \\
(\boldsymbol{\%})\end{array}$ & $\begin{array}{c}\text { TOF } \\
\left(\mathbf{h}^{\mathbf{- 1}}\right)\end{array}$ & $\begin{array}{c}\text { Selectivity } \\
(\boldsymbol{\%})\end{array}$ \\
\hline $\mathrm{PdsNC} / P N-\mathrm{CeO}_{2}$ & 27.4 & 73.6 & 10900 & 99.9 \\
$\mathrm{PdNP} / P N-\mathrm{CeO}_{2}$ & 26.3 & 38.9 & 7800 & 89.3 \\
$\mathrm{PdsNC} / P N-\mathrm{CeO}_{2}-300$ & 17.5 & 72.1 & 7968 & 99.4 \\
$\mathrm{PdsNC} / P N-\mathrm{CeO}_{2}-500$ & 13.8 & 42.5 & 6859 & 87 \\
\hline
\end{tabular}

